\title{
Recalling script-based text: The role of selective processing and schematic cues
}

\author{
FRANCIS S. BELLEZZA \\ Ohio University, Athens, Ohio
}

\begin{abstract}
Research on the learning of prose material based on scripts has attempted to relate the remembering of script events to how typical the events are of the generic scripts. The novelobject effect reported by Bellezza and Bower (1982) shows that a typical script event containing an unexpected object is rated less typical but is recalled better than an event irrelevant to the script. The novel-object effect implies that the relation between script-typicality ratings and remembering may not be a simple one. One explanation of the novel-object effect is that script sentences containing a novel object are processed longer during learning. The second explanation, the inference-contradiction explanation, suggests that the presented word representing a novel object both contradicts and yet becomes associated to information strongly inferred from the script. The results of the experiment reported here support the inferencecontradiction explanation.
\end{abstract}

Recent research has shown that organized knowledge structures, referred to here as memory schemata, play an important role in how people comprehend and remember verbal information (Rumelhart, 1980). Much of the research investigating the role of memory schemata in remembering prose-type material has concentrated on a type of schema called a script. A script deals with a stereotypical series of events involving one or more actions (Bellezza \& Bower, 1981, 1982; Bower, Black, \& Turner, 1979; Graesser, 1981; Schank \& Abelson, 1977). Graesser (Graesser \& Nakamura, 1982) has used a schema-copy-plus-tag model to explain how typical and atypical schema events, and therefore script events, are differentially recognized and recalled. In the schemacopy-plus-tag model, events in the passage typical of the activated script are copied into episodic memory. Also copied into episodic memory are inferences based on the activated script that are not in the passage at all but are very typical of the activated script. Events in the passage that do not normally occur in the script are connected to the copy of the script in episodic memory by tagged associations. In the schema-copy-plus-tag model, the schema does not filter out atypical information during comprehension of the passage, but stores and tags it in memory. Also, typical script events are not allotted additional processing during comprehension but are processed more easily because they fit the activated script.

This research was supported in part by a grant to the author from the Field-Wiltsie Foundation. The author would like to thank Kellie Vaughn and Melanie White for their help in collecting and scoring the data. Thanks also goes to Ohio University Computing and Learning Services for making computer time and their facilities available. Requests for reprints should be sent to Francis S. Bellezza, Department of Psychology, Ohio University, Athens, Ohio 45701.
A problem not dealt with by the schema-copy-plustag model results from the use made of the notion of script typicality. Typicality may not be easily represented by a unidimensional factor. Atypical information may be classified either as information that is irrelevant to a schema or information that is inconsistent with it (Hastie, 1980). In research performed with script-based texts, Bellezza and Bower (1982) made distinctions among typical script events, typical script events that include a novel object within them (inconsistent information), and atypical script events (irrelevant information). Because many types of memory schemata, including scripts, are thought to be organized in memory in a hierarchical manner, it should not be surprising that atypicality can appear on any one of a number of different levels. As an example, in the restaurant script a typical script event is "The man told the hostess that he had made a reservation." This typical event is represented by the first script event diagrammed in Figure 1. A typical script event that includes a novel person or object is "The man was taken to his table by a policeman," in which the word "policeman" replaces the more typical "hostess" or "headwaiter." A typical event

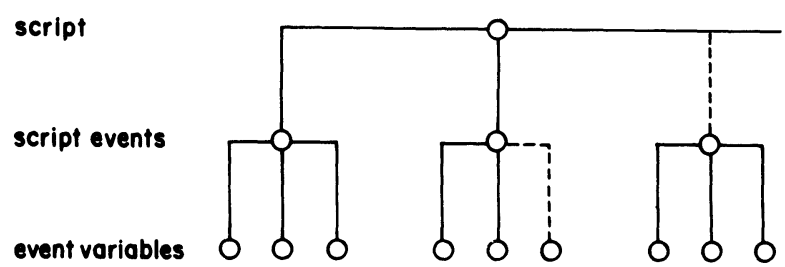

Figure 1. Three levels of hierarchical components used for a script. The dotted lines represent irrelevant events (level 2) or a novel object within a script event (level 3). (Reprinted with permission from Bellezza \& Bower, 1982; copyright 1982, by North-Holland Publishing Company.) 
containing a novel object is represented by the second script event of Figure 1. An event irrelevant to the restaurant script is "The man brushed off his shoes." This irrelevant event is represented by the third script event in Figure 1. This event, however, could be typical of some other script such as Preparing to go to work.

From the hierarchical nature of a script as diagrammed in Figure 1, it appears that typical script events containing a novel object contain more components of the script than do the irrelevant events. This means that they should be rated as more typical of the script than the irrelevant events. However, this is not what Bellezza and Bower (Experiment 1, 1982) found. Using a 6-point rating scale, subjects rated typical events as most typical (5.4), irrelevant events as the next most typical (2.9), and typical events containing a novel object as the least typical (2.0). Furthermore, a test of immediate recall showed that the typical events and the typical events containing a novel object were recalled equally well and significantly better than the irrelevant events. Thus, the relation between typicality and recall is not a straightforward one, and is a relation that is not easily explained by models such as the schema-copy-plus-tag model. According to the schema-copy-plus-tag model, the events containing a novel object and the irrelevant events should have been recalled at comparable levels because their typicality ratings were more similar to one another than they were to the ratings of the typical events. The unexpectedly good recall but low typicality ratings of script events containing a novel object will be referred to here as the novel-object effect.

The explanation Bellezza and Bower (1982) gave for the novel-object effect is based on the notion that activated memory schemata generate inferences, and that these inferences are made up of cognitive events such as images and verbalizations. Also, these cognitive events can act as cues for learning new information. When the subject reads a sentence such as "The man was taken to his table by a policeman," the segment of the sentence "The man was taken to his table by" corresponds to a typical script event in the restaurant script. Because of this, expectations are aroused in the form of images or verbalizations of the patron walking among the tables in a fancy restaurant and being led by a hostess or headwaiter. The problem is that instead of a hostess or headwaiter appearing in the sentence, as inferred by the subject, a policeman is indicated. This has two effects: First, because a specific inference can be made from the beginning of the sentence, the word "policeman" appears to be inconsistent. Therefore, the sentence as a whole is given a low typicality rating. Second, the specific inference of a man being led to his table by a hostess or headwaiter provides a set of cues generated by the script to which the word "policeman" can be associated. Later, the typical script event invoiving a man being led to his table will be likely to be recalled, and this event will act as an effective cue for the incongruous person of a policeman. Script events provide internal cues that are useful because they are inferred from the activated script both during learning and during recall, even if what is associated to the cues is not typical of the generic script. That is, these cues are constructible (Bellezza, 1981). This explanation will be referred to as the inferencecontradiction explanation of the novel-object effect. The process of associating novel information to internal cues generated by a schema also has been proposed by Hastie (1980) and Srull (1981) to explain why presented items incongruent to an activated person prototype are sometimes recalled so well.

In the case of an event irrelevant to the script, the process is different. On the event level, there are no strong expectations created as to what event should follow another event in a script-based passage. This may be because only a few script events are typically mentioned in a script-based passage, even though a large number of script events may constitute any script. The script events not explicitly mentioned are inferred. Because of this, when the subject reads an irrelevant event such as "The man brushed off his shoes," he or she usually is not expecting any particular script event. Consequently, the subject does not rate the irrelevant event as low on the rating scale as the typical event containing a novel object. Hence, a definite expectation is not unfulfilled. For the same reason, there are no specific cues being generated by the script to which the irrelevant event can be associated. As a consequence, recall of irrelevant events is poorer than recall of typical - events containing a novel object. The inference-contradiction explanation of the novel-object effect uses the script-generated inferences to explain both the low ratings of contradicted inferences and the good recall of the information contradicting the inference.

However, there is a simpler explanation of the novelobject effect. It may be that script events containing a novel object are not only rated as less typical than irrelevant events, but are also processed for a longer period of time. Therefore, they are recalled better than the irrelevant events. This is a selective-processing explanation of the novel-object effect. Atypical events have been shown to be processed longer than typical events (Bellezza \& Bower, 1981). Hastie (1980) and Srull (1981) have used this selective-processing explanation, along with the explanation that incongruous information can become associated to internal schema cues, to account for the result that traits inconsistent with an activated person prototype are remembered as well as or better than traits typical of the person prototype. These two alternative explanations of the atypical-object effect were tested.

\section{METHOD}

\section{Subjects}

Twenty-four undergraduates enrolled at Ohio University volunteered to participate for extra course credit.

\section{Materials}

Ten script-based passages, each made up of 12 events, were used. Each passage was labeled by a descriptive title that was designed to activate the appropriate script in the memory of 
the reader. One passage, The mayor gives a news conference, was always presented first as a primacy buffer, and a second passage, The little boy goes to the playground, was always presented last as a recency buffer. Only the 8 middle passages were tested for recall. The titles of these were: The salesman eats in a restaurant, The patient goes to the doctor, The wife goes to the department store, The student goes to a lecture, The banker goes on an airplane trip, The secretary works in an office, The driver goes to a gas station, and The worker gets up in the morning. The scripts used were taken from Bellezza and Bower (1982). Some of the script events were replaced by events irrelevant to the script. Also, for some of the typical script events, an object, person, or place was replaced by a novel value. More specifically, 2 of the passages remained unchanged. In 2 of the passages, an object in each of 4 of the script events was replaced by a novel object. In another 2 of the passages, 4 of the typical script events were replaced by events irrelevant to the script. In the final 2 passages, four typical objects were replaced by novel objects and 4 script events were replaced by irrelevant events. An example of this last condition is shown in Table 1 . The passages were always presented in the order given above, but each passage could be configured so that it represented one of the four conditions described above. These four conditions represented 12 typical events, 8 typical events and 4 events containing a novel object, 8 typical events and 4 irrelevant events, and 4 typical events with 4 typical events containing four novel objects and 4 irrelevant events. Four different presentation forms were used, and each passage appeared in a different condition on each form. Six subjects were tested on each form.

\section{Procedure}

The subjects were tested individually. The script passages were presented one sentence at a time on a video monitor connected to a microcomputer. The title of each passage appeared on the screen surrounded by asterisks that indicated to the subject that a new passage was starting. Presentation was self-paced by the subject. Each subject was instructed to try to understand what was happening in each passage and to be prepared to answer questions later about each passage. Each passage title and passage sentence remained on the screen until the sub-

\section{Table 1}

A Script-Based Passage Containing Four Typical Script Events, Four Irrelevant Script Events, and Four Typical Script Events Containing a Novel Object

The Banker Takes an Airplane Trip

The banker said hello to the stewardess.

He showed his shopping list to her.

(atypical object-shopping list replaces ticket)

He found his seat on the airplane.

The banker looked at the acrobats on the plane. (atypical event)

He placed his hockey stick under his seat. (aty pical object-hockey stick replaces briefcase)

He wrote awhile in his diary.

(atypical event)

The banker then fastened his seat belt.

He next turned on his sunlamp. (atypical object-sunlamp replaces reading lamp)

He chose an encyclopedia from the stewardess. (atypical object-encyclopedia replaces magazine)

The banker carefully put on his hat. (atypical event)

He ate a delicious lunch.

He then cleaned his fingernails. (aty pical event)

Note-The words in parentheses were not presented to the subjects during the experiment. ject pressed the space bar on the computer keyboard. This caused the next title or sentence to appear, and the study time was automatically recorded. After all 10 passages had been presented, the subject was given a booklet with the title of each of the 8 middle passages printed on an otherwise blank page. The subject was instructed to write down as many of the sentences as possible for each of the passages titles. The passages were tested in the same order that they were presented, and the subject was allowed as much time as needed to complete the recall task. After the recall test, the passages were again presented on the video monitor. On this presentation, the subject had to decide how likely it was that each event described by the sentence could be part of the story described by the passage. A 6-point rating scale was used. A rating of 6 meant that the event described was very likely to have occurred, and a rating of 1 meant that the event described was very unlikely. The other four values of the rating scale were also verbally labeled. The subject gave the rating by pressing one of the keys labeled 1 to 6 on the computer keyboard. The rating and decision time were automatically recorded, and the next sentence immediately appeared. The subject was instructed to press the space bar whenever a passage title appeared.

\section{RESULTS}

A sentence was scored as being recalled from a passage if the meaning of the sentence was recalled, even when the words used were different. The data from the eight passages were combined, and for each subject the following means were computed: the mean proportion recalled of typical script events, of typical events containing a novel object, and of irrelevant events. The same procedure was used to compute the mean study times for the three types of sentences, the mean decision times, and the mean typicality ratings. These means for the four measures are shown in Table 2. A repeatedmeasures analysis of variance showed the study times to be significantly different for the three types of script events $[\mathrm{F}(2,46)=5.10, \mathrm{MSe}=1.13, \mathrm{p}<.01]$. Posttests using Tukey's HSD procedure showed that the typical script events were studied for a shorter period of time than were the events containing a novel object or the irrelevant events. The means for events containing a novel object and for irrelevant events were not significantly different.

An analysis of variance on the proportion of sentences recalled showed significant differences among the three types of events $[\mathrm{F}(2,46)=6.69, \mathrm{MSe}=.008$, $\mathrm{p}<.005]$. The irrelevant events were recalled significantly more poorly than were the typical events and the typical events containing a novel object. The latter two types of sentences were recalled equally well. One problem in analyzing the recall data is that, over the eight passages, the subjects were presented a total of 64 sentences representing typical script events but only 16 sentences representing the other two types of events. The larger number of typical script events may have affected the recall levels of the three types of sentences. However, a separate analysis using only those two passages for each subject containing four sentences of each of the three sentence types showed that the subjects recalled .39 of the typical events, .38 of the events with 
Table 2

Mean Study Time (in Seconds), Proportion of Sentences

Recalled, Mean Decision Time (in Seconds), and Mean Typicality Ratings for the Three Types of Script

\begin{tabular}{lrcc}
\hline & \multicolumn{3}{c}{ Events } \\
\cline { 2 - 4 } & TE & TE with \\
& 4.40 & 5.20 & 5.32 \\
Study Time & .43 & .42 & .34 \\
Proportion Recalled & 3.09 & 3.91 & 3.71 \\
Judgment Time & 5.35 & 2.04 & 3.45 \\
\hline Mean Rating &
\end{tabular}

Note-TE = typical events; $N O=$ novel object $; I E=$ Irrelevant events.

a novel object, and .30 of the irrelevant events. This recall pattern is similar to that presented in Table 2 .

Analysis of the decision-time data again revealed a significant effect due to the type of script event $[\mathrm{F}(2,46)$ $=12.18, \mathrm{MSe}=.343, \mathrm{p}<.001]$. As in the case of study time, the mean decision time for typical script events was significantly shorter than the decision times for events containing a novel object and for irrelevant events. These latter two types of sentences were not significantly different.

Finally, analysis of the typicality ratings showed a significant effect due to sentence type $[\mathrm{F}(2,46)=81.61$, MSe $=.776, p<.001]$. Posttests showed all three means to differ significantly from one another.

\section{DISCUSSION}

The pattern of recall and typicality ratings found for the three types of script events is very similar to that found by Bellezza and Bower (1982). The typical-object effect reported by Bellezza and Bower (1982) was reproduced. The present experiment also provides data on study and decision times. As can be seen from Table 2, the patterns of mean study times and mean decision times are similar. The mean study time for events containing a novel object and the mean study time for irrelevant events were not significantly different in magnitude, but each was significantly greater than the mean study time for sentences representing typical script events. These study-time results are important because they show that the superior recall of events containing a novel object compared with the recall of irrelevant events is not the result of additional encoding time. The novel-object effect, characterized by good recall but low typicality ratings for script events containing a novel object, seems to be the result of the subject's discovering information in the passage contrary to information strongly expected. This inference-contradiction explanation of the novelobject effect was proposed by Bellezza and Bower (1982) and seems to be supported by the data reported here.
Although it cannot completely explain the novel-object effect, selective processing cannot be dismissed as a factor influencing recall in this experiment. Although the study times for novel-object sentences and irrelevant-event sentences were not significantly different, the mean study time for the novelobject sentences was significantly longer than for the typicalevent sentences. Since the novel-object sentences were recalled as well as the sentences denoting typical script events, their recall level may have been enhanced by this additional study time. Similar differences in study time have been reported by Bellezza and Bower (1981).

The importance and, indeed, necessity that knowledge structures such as memory schemata generate internal cues to which new information can be associated has been discussed in detail by Bellezza and Bower (1982). Memory schemata may reliably generate and regenerate cognitive cues to be used in the learning process in a manner similar to mnemonic devices (Bellezza, 1981; Bellezza \& Bower, 1982). The inference-contradiction explanation of the novel-object effect assumes that activated script events generate specific information that is eventually disconfirmed by what is read in the passage. Yet, somewhat paradoxically, this inferred but disconfirmed information is again generated at the time of recall and acts as a retrieval cue for information that appears in the passage but does not fit the activated script.

\section{REFERENCES}

Bellezza, F. S. Mnemonic devices: Classification, characteristics, and criteria. Review of Educational Research, 1981, 51, 247-275.

Bellezza, F. S., \& Bower, G. H. The representational and processing characteristics of scripts. Bulletin of the Psychonomic Society, 1981, 18, 1-4.

Bellezza, F. S., \& Bower, G. H. Remembering script-based text. Poetics, 1982, 11, 1-23.

Bower, G. H., Black, J. B., \& Turner, T. J. Scripts in memory for text. Cognitive Psychology, 1979, 11, 177-220.

Graesser, A. C. Prose comprehension beyond the word. New York: Springer-Verlag, 1981.

Graesser, A. C., \& Nakamura, G. V. The impact of a schema on comprehension and memory. In G. H. Bower (Ed.), The psychology of learning and motivation (Vol. 16). New York: Academic Press, 1982.

HAstie, R. Memory for behavioral information that confirms or contradicts a personality impression. In R. Hastie, T. M. Ostrom, E. B. Ebbesen, R. S. Wyer, D. L. Hamilton, \& D. E. Carlston (Eds.), Person memory: The cognitive basis of social perception. Hillsdale, N.J: Erlbaum, 1980.

RUMELHART, D. E. Schemata: The building blocks of cognition. In R. Spiro, B. Bruce, \& W. Brewer (Eds.), Theoretical issues in reading comprehension. Hillsdale, N. J: Erlbaum, 1980.

Schank, R. C., \& Abelson, R. P. Scripts, plans, goals, and understanding. Hillsdale, N. J: Erlbaum, 1977.

SRULl, T. Person memory: Some tests of associative storage and retrieval models. Journal of Experimental Psychology: Human memory and learning. 1981, 7, 440-463.

(Manuscript received for publication May 2, 1983.) 